\title{
Cultura de reciclaje en México: La educación ambiental
}

\author{
Recycling culture in México: Environmental education
}

\author{
Adriana N. Velázquez-García ${ }^{a}$, Alexandra Trejo-Sánchez ${ }^{b}$, Jesús B. Tobón-García $^{c}$
}

\begin{abstract}
:
At the global level there is a discussion about environmental disasters, which are occurring more and more frequently due to human activities and lack of respect for their ecosystems, to such an extent that today the whole world is facing an environmental crisis and not just climate problems. Faced with this situation, the "Culture of Recycling" represents an alternative for the planet to carry out its restoration processes in the face of the large amount of waste generated over time. Countries are responsible for implementing this culture in different areas, through regulations, education and the creation of organizations to support this new way of life.
\end{abstract}

Keywords:

Environmental problems, ecosystem, recycling culture, waste, environmental education

\section{Resumen:}

A nivel mundial existe una discusión sobre las catástrofes ambientales, que cada vez ocurren con mayor frecuencia debido a las actividades humanas y la falta de respeto a sus ecosistemas, a tal grado que hoy en día, el mundo entero está enfrentándose a una crisis ambiental y no a simples problemas climáticos. Ante esta situación, la "Cultura de Reciclaje" representa una alternativa para que el planeta lleve a cabo sus procesos de restauración frente a la gran cantidad de residuos generados al transcurrir del tiempo, siendo responsabilidad de los países la implementación de esta cultura en diferentes ámbitos, a través de la normatividad, la educación y la creación de organizaciones de apoyo a esta nueva forma de vida.

\section{Palabras Clave:}

Problemas ambientales, ecosistema, cultura de reciclaje, residuos, educación ambiental

\section{Introducción}

Una de las problemáticas más severas a que se enfrenta la mayoría de países alrededor del mundo, es el incremento en la generación de residuos sólidos y la contaminación que éstos producen, lo que ha dado lugar a que dentro de las agendas de los gobiernos se implementen acciones para su manejo, disposición y contribución al cuidado del medio ambiente (Sanmartín , Zhigue, \& Alaña, 2017) mediante el reciclaje.

Estas acciones no son nuevas, la literatura refiere que el reciclaje surgió desde las primeras civilizaciones, con el asentamiento de grupos humanos que se vieron precisados a reutilizar sus materiales y la "basura" generada por sus actividades (LYRSA, 2015) cuando, tras la aparición y uso del fuego, este problema se complicó. Con el invento del papel y su reutilización en la elaboración de pergaminos y papiros en Japón y Egipto, primeros países en reciclar, el concepto de "basura" cambia por el de "residuos sólidos", encontrándose en la actualidad la diferencia entre estos dos conceptos en la Ley General para la Prevención y Gestión Integral de los Residuos, Titulo Primero, articulo 5, fracción XXIX, que define como "residuo sólido" a todo material que puede

\footnotetext{
a Escuela Superior de Tizayuca, Universidad Autónoma del Estado de Hidalgo, México, Km. 2.5. Carretera Federal Tizay uca - Pachu ca, 43802, Tizayuca, Hidalgo.https://orcid.org/0000-0002-2447-7386,email: ve312922@uaeh.edu.mx

b Escuela Superior de Tizayuca, Universidad Autónoma del Estado de Hidalgo, México, Km. 2.5. Carretera Federal Tizay u ca - Pachu ca, 43802, Tizayuca, Hidalgo.https://orcid.org/0000-0002-4240-3567, email: tr339378@uaeh.edu.mx

c Escuela Superior de Tizayuca, Universidad Autónoma del Estado de Hidalgo, México, Km. 2.5. Carretera Federal Tizay uca - Pachuca, 43802, Tizayuca, Hidalgo.https://orcid.org/0000-0003-1578-893X, email: to362600@uaeh.edu.mx
} 
ser susceptible a ser valorizado, mientras que la "basura" es todo aquello que ya no tiene utilidad y por tanto, no puede ser reciclado o reutilizado (SEMARNAT, 2003). Los procesos de transformación económica, social y tecnológica en la segunda mitad del siglo XVIII, Ilevaron al hombre a la recuperación de prendas de ropa, metales, piedras y otros materiales durante la revolución industrial, de tal forma que la época subsecuente fue bautizada por diversos historiadores como "la edad de oro del reciclaje". Durante la segunda guerra mundial, la práctica del reciclaje se convirtió en una actividad fundamental para la sobrevivencia, ya que en momen tos de crisis la reutilización o venta de los materiales que se tenían en casa permitió a los individuos subsistir económicamente (Arenas, 2016), no obstante, fue hasta el siglo XXI que la cultura de reciclaje empezó a tener impacto en función de la contaminación originada por los residuos que se han generado con el paso de los años, llegando al grado de amenazar con la destrucción de la vida humana, en un futuro cercano.

La tendencia al reciclaje a nivel mundial se observa en los datos del Banco Mundial (BM), que refiere a países eu ropeos como Suiza, Suecia, Austria, Alemania, Bélgica y los Países Bajos como aquellos con mayores indicadores de reciclaje, dado que sus niveles superando el $50 \%$ total de sus desechos anuales; mientras que América Latina es la región menos comprometida en esta actividad, señalando a México como el país que genera mayor cantidad diaria de residuos sólidos (Montes, 2019).

Ante esta situación, México se ha comprometido a fomentar la cultura de reciclaje mediante actividades para reciclar el plástico de tereftalato de polietileno (PET por sus siglas en Inglés), buscando posicionarse en un nivel similar al de los países europeos, y asumiendo el liderazgo en América Latina, con una tasa del $56 \%$ en actividades de acopio y reciclaje, que representa casi el doble de la tasa de reciclaje de Estados Unidos (EI Universal, 2019). Destacándose las Ciudades de México (CDMX), Monterrey y Guadalajara, como las localidades mexicanas que más reciclan (INEGI, 2015).

En función de lo anterior, el propósito de este artículo es exponer aspectos representativos que evidencien una cultura de reciclaje en las ciudades mencionadas, consideradas como zonas con mayores actividades de reciclaje, gracias a la preocupación por el control de sus altos índices de contaminación. Para lograr dicho propósito se procedió a realizar el análisis y comparación de aspectos básicos requeridos para orientar una adecuada cultura de reciclaje, como son la normatividad, la educación ambiental y la existencia de organizaciones que apoyan al fortalecimiento de la cultura objeto de estudio en este artículo.

\section{Normatividad que apoya a la cultura de reciclaje en la CDMX, Monterrey y Guadalajara.}

El punto de partida se encuentra en la realización de un análisis de la normatividad vigente para cada una de estas ciudades, debido a la importancia que tiene en la cultura de reciclaje en dos aspectos fundamentales: a) constituye la base para coordinar el reciclaje y tratamiento de residuos sólidos, y b) contribuye a evitar conductas negativas perjudiciales para el medio ambiente y la salud.

En la Ciudad de México (CDMX) se contemplan las siguientes normativas:

1. Ley de Residuos Sólidos del Distrito Federal, publicada el 22 de Abril del 2003, con una última reforma en el año 2019. Su propósito es el de regular la gestión integral de residuos sólidos considerados como no peligrosos en el Distrito Federal (LEGISMEX, 2019)

2. Reglamento de la Ley de Residuos Sólidos del Distrito Federal, que tiene por objeto reglamentar dicha ley en materia de gestión integral de residuos sólidos no peligrosos y servicio de limpia, integrando en el documento las definiciones contenidas en esta ley. Este reglamento fue publicado el 7 de Octubre del 2008, conformado de 9 títulos, destacando por su alta relación con el tema principal de este artículo el "Título Segundo: De la Política Ambiental", que expone los diversos programas que se han realizado en relación a los residuos sólidos, así como, las normas ambientales para el Distrito Federal. Impone también que la cultura ambiental es una materia que debe incorporarse en todos los niveles educativos.

En otro de sus apartados, el "Titulo tercero: de la prevención y minimización de la generación de los residuos sólidos", establece la clasificación de los residuos sólidos, el plan de manejo de los mismos que los generadores de residuos están obligados a presentar, y la separación adecuada de estos desechos.

Por otra parte, el "Título cuarto: Del servicio público de limpia" enfatiza la recolección, transferencia y tratamien to de los residuos sólidos.

En el año de 2020, con fecha 2 de enero, la Jefa de Gobierno llevó a cabo la modificación del mencionado reglamento, con el fin de mejorar los mecanismos que permitan cumplir con el compromiso asumido en este rubro; debido a que la CDMX es una de las ciudades más grandes del mundo y por lo cual en ella se genera 
una gran cantidad de residuos sólidos (LEGISMEX, 2008)

3.- Se contempla también la Norma Ambiental de Separación de Residuos (NADF-024-AMBT-2013), emitida por el Gobierno a través de la Secretaría del Medio Ambiente, que entró en vigor en Julio 2017, cu yo principal objetivo consiste en regular las actividades para reducir la cantidad de basura que ingresa a los rellenos sanitarios, así como incrementar el reciclaje de los residuos sólidos.

En dicha norma se establecen los criterios para realizar una adecuada selección, clasificación (orgánicos, manejo especial y voluminoso, inorgánicos reciclables, e inorgánicos no reciclables) y valorización de los residuos sólidos. Colocando a la CDMX en el mismo nivel de ciudades de Latinoamérica como Buenos Aires, Bogotá, Santiago, Lima y Quito; así como París, Berlín, Los Ángeles y Nueva York, donde ya se aplica una política de separación para la reutilización de los residuos sólidos (Sedema, 2018).

En lo que respecta a la ciudad de Monterrey, tiene aplicación:

1. Ley Ambiental del Estado de Nuevo León, cuya última reforma fue publicada el 29 de Enero del 2020 (Congreso del Estado de Nuevo León, 2020), que de acuerdo al Artículo I del capítulo I "Normas Preliminares", expresa lo siguiente: "la presente ley es reglamentaria del segundo párrafo del artículo 3 de la Constitución Política del Estado de Nuevo León, sus disposiciones son de orden público e interés social, y tienen por objeto propiciar la conservación y restauración del equilibrio ecológico, la protección al ambiente y el desarrollo sustentable del Estado".

De igual forma, el capítulo V "Manejo y Gestión Integral de los Residuos" del título IV "Protección al Ambiente", establece en su Artículo 169 las atribuciones que en materia de residuos ejercerán las autoridades señaladas por la ley, en tanto que el Artículo 180 refiere las medidas, técnicas y procedimientos que las autoridades normalizarán y promoverán para la separación, clasificación, reúso y reciclaje de los residuos, señalando también los materiales que permitan reducir la contaminación al ambiente.

2. Reglamento de la Ley Ambiental del Estado de Nuevo León, publicado el viernes 29 de febrero del 2008, con una última reforma publicada el 29 de Agosto del año 2016, y que establece los procedimientos para la emisión de las autorizaciones que se señalan.
La ley mencionada otorga, en su artículo 8, la facultad que la Agencia de Protección al Medio Ambiente y Recursos Naturales tiene para ser la autoridad encargada de la aplicación del Reglamento, promoviéndolo entre los municipios que forman la zona conurbada de Monterrey, Nuevo León (LEGISMEX, 2019).

3. Norma Ambiental Estatal (NAE-SDS-005-2019). Publicada en el Periódico Oficial del Estado el 15 de Julio del 2005 y su reforma posterior publicada con fecha 10 de Mayo del año 2019, en el cual se reforma por modificación el artículo 168-Bis de la Ley Ambiental del Estado de Nuevo León. Su propósito es establecer las especificaciones y métodos para determinar el porcentaje de material reciclado, y el uso de materiales de ágil degradación en las bolsas de plásticos, en el Estado de Nuevo León (Moya, 2019).

En cuanto a la normatividad aplicable en la ciudad de Guadalajara se contempla lo siguiente:

1. Ley de Gestión Integral de los Residuos del Estado de Jalisco, cuya última reforma fue publicada en el Periódico Oficial el 27 de Abril del año 2019.

Esta ley consta de siete títulos y 99 artículos, más transitorios; sus objetivos son:

I. Establecer las Políticas Públicas en Materia de Gestión de Residuos en el Estado; II. Promover el establecimiento de medidas que prevengan el deterioro de los ecosistemas en el manejo y disposición final de residuos; III. Establecer las bases para la participación ciudadana en la reutilización y manejo de residuos; IV. Establecer la competencia concurrente entre la Federación y el Estado. V. Involucrar a los generadores de residuos; VI. Garantizar el derecho a toda persona al medio ambiente adecuado y propiciar el desarrollo sustentable; VII. Establecer mecanismos de coordinación entre el Estado y los Municipios; VIII. Controlar y prevenir la contaminación y remediación de áreas contaminadas; IX. Fomentar la reutilización y valorización de los materiales contenidos en los residuos que se generan en el Estado; y X. Garantizar el cumplimiento de esta ley y las disposiciones que de ella emanen (Gobierno, 2019).

2. Norma Ambiental Estatal NAE-SEMADES007/2008, publicada en el Periódico Oficial el 16 de Octubre del año 2008, con el propósito de establecer los criterios y especificaciones técnicas bajo las cuales se deberá realizar la separación, clasificación, recolección selectiva y valorización de los residuos en el Estado de Jalisco, con lo cual se promoverá el establecimiento de 
medidas que prevengan el deterioro de los ecosistemas por el inadecuado manejo y disposición final de residuos, reconociendo la responsabilidad compartida de todos los actores involucrados y estableciendo las bases para la participación ciudadana en la gestión integral de residuos (González Márquez, Guzmán Pérez, \& Castell Castillo, 2008)

3. Reglamento de la Ley de Gestión Integral de los Residuos del Estado de Jalisco, expedido el viernes 1 de Julio del año 2011 y publicado el sábado 3 de Agosto del mismo año, con el objetivo de ser el instrumento normativo que coadyuve al establecimiento de una política estatal en materia de Gestión Integral de Residuos, en términos de los establecidos por la Ley de la Gestión Integral de los Residuos, para brindar así un manejo eficiente de los mismos, que contribuyan a mejorar las condiciones ambientales.

Para el cumplimiento del reglamento referido se contempla observar los principios que se establecen en la Ley General para la Prevención y Gestión Integral de los Residuos, la Ley de Gestión Integral de Residuos del Estado de Jalisco y demás disposiciones aplicables (LEGISMEX, 2011).

4. Reglamento para la Prevención y Gestión Integral de los Residuos del Municipio de Guadalajara, que de acuerdo a su artículo 1 establece que es de observancia general en el Municipio de Guadalajara, Jalisco; sus disposiciones son de orden público e interés social y tiene por objeto regular la prevención de la generación y la gestión integral de los residuos sólidos urbanos, de manejo especial y peligrosos (cuando corresponda a los micro generadores o generados en casas habitación) que no estén expresamente atribu idos a la Federación o a la entidad federativa, de conformidad con lo que establece la Ley General para la Prevención y Gestión Integral de los Residuos y la Ley de Gestión Integral de los Residuos del Estado de Jalisco; así como regular y organizar la prestación del servicio de aseo público municipal.

En el título tercero "Del Manejo Integral de Residuos", se implementa desde la prevención y minimización de la generación de residuos hasta la recolección, tran sporte, transferencia, valorización y tratamiento de residuos.

Es importante aquí mencionar que el capítulo XIV "Del reciclaje de residuos", en su Artículo 85 establece que "el Ayuntamiento debe fomentar programas para redu cir la generación de residuos, aprovechar su valor y darles un manejo ambientalmente adecuado, en los establecimientos de mayoristas, tiendas de departamentos y centros comerciales, donde se cuente con espacios y servicios destinados a la recepción de materiales y subproductos de los residuos sólidos valorizables" (LEGISMEX, 2016).

Como puede observarse, la ciudad de Guadalajara es la que brinda mayor apoyo a la cultura de reciclaje en el ámbito normativo, ya que en esta zona se cuenta con dos reglamentos específicos para el manejo de residuos, además de la ley y su norma referidas, a diferencia de la ciudad de Monterrey, donde únicamente se considera este aspecto de forma general, conforme a lo señalado en algunas fracciones, en relación a regulaciones exclusivas para el reciclaje de residuos.

Conviene también destacar que la Ciudad de México cuenta con una norma específica para la separación de sus residuos, a diferencia de lo establecido en la normatividad aplicable en las otras dos ciudades analizadas; lo que lleva a sus ciudadanos a mejores prácticas de reciclaje, en beneficio del medio ambiente y la salud humana, aspecto significativo, ya que como se sabe, la CDMX es la ciudad más contaminada en el país, debido al incremento en la urbanización registrado en los últimos años.

Finalmente, debe señalarse que en cuanto al man ejo de residuos existe una misma regulación para estas tres ciudades, ya que a nivel nacional se ha establecido la Ley General para la Prevención y Gestión integral de los Residuos; normatividad reglamentaria de acuerdo a las disposiciones de la Constitución Política de los Estados Unidos Mexicanos, y que hace referencia a la protección del ambiente mediante la prevención y gestión integral de residuos (Diario Oficial de la Federación, 2018).

Para dar continuidad al análisis planteado en este trabajo, se presentan a continuación aspectos comparativos relacionados a la Educación Ambiental en las tres ciudades implicadas.

\section{Educación ambiental}

\section{Ciudad de México (CDMX)}

Considerando en primera instancia la CDMX, se hace la observación de que en ella existen Centros de Educación Ambiental (CEA), denominados también Centro de Cultura Ambiental (CCA), donde se imparten diversos programas, implementados con el propósito de concientizar a la ciudadanía sobre la importancia del reciclaje y cuidado del medio ambiente, creados en su mayoría por la Secretaría del Medio Ambiente del Gobierno de México Distrito Federal (SEDEMA, 2020). Estos centros son los siguientes: 
- $\quad$ Acuexcómatl, fundado en 1998 en la Alcaldía Xochimilco, y acreditado en el periodo 2011-2012 con la categoría de "espacio comprometido con la educación ambiental" que otorga la SEMARNAT.

- Ecoguardas, establecido desde 1986 en la Alcaldía Tlalpan, cuenta hoy en día con el mismo reconocimiento que el CEA Acuexcómatl.

- $\quad$ Yahutlica, que opera desde el año de 1999 en la Alcaldía Iztapalapa.

Como ya se mencionó, el objetivo de estos centros es motivar a la sociedad a proteger sus recursos naturales mediante talleres de reciclaje, ecotecnias y juegos al aire libre. Dirigidos especialmente al público infantil, se fundamentan en los principios y valores de la Institución, que contribuyen a la construcción de una sociedad más sustentable y participativa (SEDEMA, 2017).

Entre los programas que se imparten en los CEA se contemplan los siguientes:

Programa Escolar de separación para el reciclaje de residuos: Implementado en escuelas primarias de la Ciudad de México con el propósito de fomentar la cultura de manejo adecuado de los residuos sólidos, a través de la correcta separación para llevar a cabo el reciclaje. Este programa tiene impacto tanto en las escuelas como en los hogares de cada estudiante, ya que está dirigido no únicamente a los niños, sino también a los docentes y padres de familia. Entre sus actividades se contemplan talleres de capacitación, emisión de folletos y realización de ferias de educación ambiental ( CENEA, 2019).

Programa de Educación Ambiental ItineranteEDUCABIT (PEAI), dirigido especialmente a personas que no pueden asistir a los CEA. Su estrategia educativa consiste en asistir a diversos espacios como escuelas, plazas, parques deportivos y empresas para acercarse a la ciudadanía, sensibilizarlos y capacitarlos, considerando que el contacto con las personas y sus problemáticas ambientales dentro de dichos espacios permite trabajar y aprender al mismo tiempo de las experiencias y preocupaciones de cada uno.

De este último programa se derivan dos programas más: a) Aprendiendo por el bosque y b) Ambiente de plaza. EI primero de ellos fue implementado en el Bosque de Chapultepec, debido al progresivo deterioro sufrido por las acciones negativas de sus visitantes, que provocaron eutrofización de los lagos, pérdida de especies, maltrato a las áreas verdes y dispersión de residuos sólidos. Su objetivo es favorecer su conservación e impulsar una cultura ambiental, a través de visitas, actividades educativas y talleres ambientales, así como la formación de educadores ambientales en espacios públicos y el reconocimiento de un gran número de instituciones, escuelas y personas.

El segundo programa derivado, "Ambiente de plaza", busca la participación social mediante acciones educativas orientadas al mejoramiento ambiental de las Plazas Públicas, a través de la integración y operación de grupos de voluntarios (CENEA, 2019).

\section{Monterrey}

En lo que respecta a la ciudad de Monterrey, debe destacarse la importancia que se da a la educación ambiental no sólo en la ciudad capital, sino a nivel estatal, señalándose que en el año de 2017 fue presentada una iniciativa de reforma a la Ley Estatal de Educación con el objeto de impulsar el reciclaje de los residuos sólidos, así como la implementación de contenedores para la separación de basura en las escuelas, en todos los niveles educativos, a través de campañas de recolección de PET, vidrios, papel, alu minio, cartón y residuos orgánicos (Dóriga, 2017).

Es así entonces que el Tecnológico de Monterrey, institución educativa que goza de gran prestigio a nivel nacional, se unió al fortalecimiento de la educación ambiental con el despliegue de un programa a cargo de su cuerpo de investigadores en el municipio de Santa Catarina, N.L., y que se ha extendido hasta municipios de estados aledaños (ITESM, 2019).

De igual forma, ha implementado acciones importantes para promover el reciclaje entre su comunidad, apoyándose con la instalación de contenedores en puntos estratégicos, motivando a los estudiantes y trabajadores a depositar en ellos los desechos de papel, aluminio, plástico y materiales orgánicos, entre otros.

Las acciones mencionadas están presentes no solamente en el campus de la ciudad de Monterrey, sino en la totalidad de sus instalaciones en el país, en don de los alumnos y colaboradores tienen la libertad de depositar en los contenedores, materiales traídos desde sus casas, incluyendo basura electrónica, para su correcto desecho o reutilización (Tamayo, 2018).

Como se observa, desde la ciudad capital del estado de Nuevo León a través de una institución educativa de prestigio se promueven acciones para generar un impacto social en materia de educación ambiental.

\section{Guadalajara}

En cuanto a las acciones de educación ambiental que se encuentran presentes en la ciudad de Guadalajara y de manera general en el estado de Jalisco, la cultura de 
reciclaje principalmente de plásticos, es promovida en tre la comunidad de la Universidad Autónoma de Guadalajara (UAG), así como la "sustentabilidad como modelo educativo" con el p ropósito de contribuir a que se genere una cultura para llevar a cabo acciones que beneficien al planeta (El Universal, 2019).

En otros niveles educativos, desde preescolar hasta bachillerato, también se fomenta el reciclaje con resultados significativos más allá del ámbito escolar. Ejemplo de esto es el "Eco Reto", programa de acopio para promover la correcta gestión de residuos y empaques post-consumo a cargo de "Ecología y Compromiso Empresarial” (ECOCE, A.C.), asociación ambiental sin fines de lucro, comprometida para la construcción de un futuro sustentable mediante la creación de nuevos hábitos en el manejo de residuos generados en los hogares y escuelas (ECOCE, 2020).

En el estado de Jalisco, esta asociación llevó a cabo el Eco Reto del ciclo escolar 2016-2017, en el que participaron 80 escuelas y más de 30 mil alumnos de Guadalajara y otros municipios jaliscienses (Razo, 2017) Durante el periodo señalado se logró el acopio de casi 40 toneladas de PET para ser recicladas, lo que dio lugar al reconocimiento y premiación a las escuelas participantes, por parte de la asociación ambiental promotora.

Lo anterior es una evidencia clara de que la educación ambiental, más que limitarse a un aspecto concreto del proceso educativo, debe convertirse en una base para elaborar un nuevo estilo de vida; en una práctica educativa abierta a la vida social, con el propósito de que los miembros de la sociedad participen, según sus posibilidades, en la tarea compleja y solidaria de mejorar las relaciones entre la humanidad y su medio (Huerta, 2019).

\section{Del dicho al hecho. Las organizaciones y el cuidado ambiental}

Hablar de la creación de una cultura ambiental nos conduce a la reflexión acerca de que este es un proceso que va más allá de plantar árboles o hacer conciencia de los residuos que generamos; implica tener conocimiento de lo que es basura y lo que es susceptible de ser reciclado; sugiere cuestionarse sobre la separación adecuada de los residuos, el proceso de reforestación de los bosques y otros aspectos presentes tanto en los hogares como en las grandes empresas, para adquirir conocimientos sobre el cuidado del ambiente.

Es en este punto donde debe reconocerse la existencia de diversas normativas en materia ambiental, que contribuyen para regular las actividades empresariales, conservar los recursos y el ambiente, y contribuir a que el mundo siga siendo un lugar habitable.

En este orden de ideas y gracias a la influencia internacional, en diversos ámbitos de acción en México ha sido aceptado un mecanismo de evaluación de índices ambientales. En particular, a partir de la formulación de la Ley General de Equilibrio Ecológico y Protección Ambiental (LGEEPA) en 1988, se crearon diversas instituciones con sentido de protección ambiental que contemplan la evaluación de impacto y riesgo ambiental dentro de sus tareas, como la Secretaría de Medio Ambiente y Recursos Naturales (SEMARNAT) y el Instituto Nacional de Ecología (INE) en el ámbito federal (INE-Semarnap, 2000b), y la Secretaría del Medio Ambiente del Gobierno del Distrito Federal, en el nivel estatal (GDF, 2008).

Es así entonces que cada vez se cuenta con mayor cantidad de empresas que más allá de buscar incrementar su cantidad de clientes mediante el fomento de tendencias como el "consumismo Green", han aportado a la conservación del ambiente, y entrado, a la par, a nuevos segmentos.

A continuación, se hace referencia a empresas y organizaciones que de alguna forma han contribuido a mejorar el ambiente y que en México pueden conformarse como ejemplo para otras empresas $u$ organizaciones, así como coadyuvante para la adh esión a la legislación vigente y la adopción de medidas de mejora en distintos ámbitos.

Si bien algunas de estas organizaciones son reconocidas y visibles a nivel nacional, existen otras de las cuales se desconoce su propósito y actividades. Claro ejemplo es ECOCE A.C., que como fue mencionado en apartados anteriores, es una organización ambiental sin fines de lucro que además de contribuir a la creación de hábitos para manejo de residuos, ha adoptado un compromiso social relevante, orientado a la construcción de un futuro sustentable. Esta asociación enfatiza la falta de educación ambiental en México, por lo que contribuye a propiciar el reciclaje y la adecuada separación de los residuos.

La casi nula educación ambiental en el país complica el ejercicio y la creación de una cultura de reciclaje, no obstante, en México están establecidas empresas visibles a nivel mundial, que contribuyen a la conservación del ambiente, como es el caso de CocaCola, pionera en la implementación del uso de botellas retornables, promotora de la separación de desechos como primer paso para la reutilización de materiales 
(Coca-Cola, 2020) y participante de iniciativas como el "Acuerdo Nacional para la nueva economía del plástico" y "Un mundo sin residuos", este último de manera conjunta con otras embotelladoras mexicanas.

Así mismo y aún después de muchos años, esta empresa continúa creando campañas dirigidas a la sociedad en general, orientadas a la reutilización, creación de conciencia en temas ambientales y compromiso con el planeta.

Otra de las empresas establecidas en México que contribuyen a minimizar los efectos medioambientales derivados de las actividades empresariales es Nestlé; mediante su principio de "Creación de valor compartido" integrado a sus acciones, despliega objetivos de desarrollo rural para el cultivo saludable, responsable y sostenible por parte de sus proveedores agricultores, así como para la protección del agua y acciones para fren ar el cambio climático y salvaguardar el medio ambiente (Ramírez, 2018).

La lista de empresas responsables en México, en aspectos de gestión medioambiental es larga e inclu ye a Hewlett-Packard con el adecuado manejo de residuos, especialmente basura electrónica; CuauhtémocMoctezuma con énfasis en el manejo del medio ambiente; así como Walmart como administrador eficaz del medio ambiente con alta eficiencia ecológica y energía proveniente de fuentes renovables en un $25 \%$, entre otras (Expansión, 2014). Otros ejemplos son Grupo Bimbo, caracterizado por la conversión de unidades con ciclo de vida a punto de concluir, en vehículos eléctricos; embotelladora Femsa, que hace uso de biocombustible que genera vapor y ahorra combustible; Cemex, cuyo consumo eléctrico proviene de energía renovable y Soriana, que se abastece de energía eólica, evidenciando su contribución para reducir el impacto ambiental (Expansión, 2015).

Las acciones que estas empresas realizan, definitivamente son un impulso a favor de la ecología, dejando en claro que contar con modelos de gestión ambiental, además de propiciar el cuidado el ambiente contribuye a que se incremente su posicionamiento en el mercado y en consecuencia, el número de clientes y sus ingresos por ventas; no obstante, lo más relevante es que constituyen un ejemplo a seguir, en cuanto a la mejora en aspectos ambientales y la preservación del país, así como para crear esa cultura tan anhelada del reciclaje y de esa forma, generar una vida digna para futuras generaciones.

\section{Conclusiones}

Hablar de educación ambiental y de la creación de una cultura de reciclaje va más allá de la educación tradicional en un salón de clases. Si se hace referencia al simple proceso de impartir el conocimiento y expan dirlo, debe considerarse que es necesario relacionar la contaminación ambiental con el ser humano y su entorno inmediato, y propiciar acciones que contribuyan a tomar conciencia real sobre las diferentes alternativas de apoyo a la conservación de su medio y su sustentabilidad, de tal forma que se eliminen las alteraciones en las cadenas alimenticias y se mantenga la calidad de vida.

No obstante que el reciclaje se ha convertido en un hábito consciente y el apoyo para su práctica proviene de campañas, programas gubernamentales e incluso de iniciativas pedagógicas, se hace necesario ampliar la información acerca de sus beneficios e incidir con mayor fuerza en los procesos educativos de la población desde los niveles básicos, dado que el impacto que se genera en los estudiantes, alcanza sus hogares y a la sociedad en general.

Se considera que si se inculca una cultura ambientalista desde edades tempranas, se estará empezando la formación de ciudadanos responsables y comprometidos con el medio ambiente, dando lugar a que las leyes, normas y reglamentos emitidos para la regulación en el manejo de residuos sólidos y la reutilización de materiales actúen como guías orientadoras de las prácticas de la sociedad y las empresas, no como elementos punitivos para sancionar comportamientos $y$ prácticas no adecuadas que perjudican tanto al medioambiente como a la salud humana.

Si bien las instituciones educativas en México todavía no son sujetos obligados a la adhesión de modelos de educación ambiental como en otros países, es claro que en los estudiantes de diferentes niveles se está empezando a propiciar el desarrollo de una conciencia ecológica, para mitigar los efectos del cambio climático, la contaminación y el deterioro medioambiental, a través de esquemas que promueven acciones para el restablecimiento del equilibrio ecológico.

Se observa también un mayor compromiso en las empresas, que incluso han generado cambios en sus productos, elaborándolos a partir de materias primas y con materiales amigables con el medio ambiente, incorporando a sus procesos el uso de tecnologías y energía de mayor eficiencia y menor impacto ambiental, disponiendo adecuadamente de sus residuos sólidos y procurando la certificación de sus procesos y prácticas 
bajo estándares de calidad establecidos por las normas de la familia ISO, NOM y NMX.

En esencia, la creación de un cultura de cuidado del ambiente implica no sólo el conocimiento y adhesión a la normativa vigente sobre manejo de residuos sólidos y reutilización o reciclaje de materiales, sino más bien requiere de la adopción de actitudes conscientes, la aceptación de responsabilidades sociales y ambien tales que generen cambios reales en el mundo, la buena relación humano-ambiente, la preservación de los ecosistemas, la observancia de principios éticos para el cuidado y conservación del planeta, y la inclusión de cuestiones ambientales en los procesos formativos de los estudiantes en los diferentes niveles educativos, camino que en México ya se ha empezado a recorrer.

\section{Referencias}

CENEA. (8 de Noviembre de 2019). México. Secretaría del Medio Ambiente del Gobierno de México Distrito Federal, Dirección de Educación Ambiental (DEA). Obtenido de Centro Nacional de Educación Ambiental - CENEAMicono barra herramientas: https://webcache.googleusercontent.com/search?q=cache:6Yon HNyvBQ8J:https://www.miteco.gob.es/es/ceneam/recursos/qui en-es-quien/mexicodf-

$\mathrm{dea} \cdot \mathrm{a} \mathrm{sp}+\& \mathrm{~cd}=11 \& \mathrm{hl}=\mathrm{es} \& \mathrm{ct}=\mathrm{clnk} \& \mathrm{gl}=\mathrm{mx}$

Arenas, M. (11 de Noviembre de 2016). Reciclaje, todo comenzó el día que los griegos descubrieron los vertederos. Obtenido de Ferrovial: https://blog.ferrovial.com/es/2016/11/reciclajecomenzo-cuando-los-griegos-descubrieron-los-vertederos/

Coca-Cola. (2020). Medio ambiente. Obtenido de Coca Cola México: https://www.coca-colamexico.com.mx/temas/medio-ambiente

Congreso del Estado de Nuevo León. (29 de Enero de 2020). Ley Ambiental del Estado de Nuevo León. Obtenido de Leyes: http://www.hcnl.gob.mx/trabajo_legislativo/leyes/leyes/ley_am biental_del_estado_de_nuevo_leon/

Diario Oficial de la Federación. (19 de Enero de 2018). Ley General para la Prevención y Gestión Integral de los Residuos. Obtenido de Camara de Diputados: http://www.diputados.gob.mx/LeyesBiblio/pdf/263_190118.pd $\mathrm{f}$

Dóriga, L. (8 de Noviembre de 2017). Impulsan legislación para reciclaje de sólidos en escuelas de Nuevo León. López Dóriga Obtenido de https://lopezdoriga.com/nacional/impulsanlegislacion-para-reciclaje-de-solidos-en-escuelas-de-nuevoleon/

ECOCE. (2020). Economía y Compromiso Empresarial. Obtenido de https://www.ecoce.mx/

El Universal. (25 de Noviembre de 2019). México, líder de acopio y reciclaje de plástico PET. El Universal. Obtenido de https://www.eluniversal.com.mx/nacion/mexico-lider-deacopio-y-reciclaje-de-pla stico-pet

El Universal. (8 de Agosto de 2019). Promueven cultura del reciclaje en la UAG. Unión Jalisco. Obtenido de https://www.unionjalisco.mx/articulo/2019/08/08/educacion/pr omueven-cultura-del-reciclaje-en-la-uag

Expansión. (23 de diciembre de 2014). Las 10 empresas más responsables en México. Obtenido de Expansión https://expansion.mx/negocios/2014/12/16/las-10-empresasmas-responsables-en-mexico

Expansión. (10 de junio de 2015). 8 proyectos de empresas amigables con el medio ambiente. Obtenido de Expok: https://www.expoknews.com/8-proyectos-de-empresasamigables-con-el-medio-ambiente/
Gobierno, S. G. (27 de Abril de 2019). Ley de Gestiòn Integral de los Residuos del Estado de Jalisco. Obtenido de Leyes: https://info.jalisco.gob.mx/sites/default/files/leyes/ley_de_gesti on_integral_de_los_residuos_del_estado_de_jalisco.pdf

González Márquez, E., Guzmán Pérez,F. A., \& Castell Castillo, J. M. (16 de Octubre de 2008). Norma Ambiental Estatal NAESEMADES-007/2008. Obtenido de Periódico Oficial del Estado de Jalisco : http://legismex.mty.itesm.mx/estados/ley-jal/JALNAE-SEMADES007_2008.pdf

Huerta, J. F. (Mazro de 2019). Fundamentos de la Educación Ambiental. Obtenido de Manual Educación Medio Ambiente: https://webcache.googleusercontent.com/search?q=cache:gWm Z3f2q-

2cJ:https://www.unescoetxea.org/ext/manual/html/fundamento $\mathrm{s} . \mathrm{html}+\& \mathrm{~cd}=1 \& \mathrm{hl}=\mathrm{es} \& \mathrm{ct}=\mathrm{clnk} \& \mathrm{gl}=\mathrm{mx}$

INEGI. (5 de Abril de 2015). Estadísticas Ambientales. Obtenido de SEMARNAT:

https://www.gob.mx/cms/uploads/attachment/file/323492/CTE IERSP_Sesio_n_abril_5_2018__RESIDUOS_SOLIDOS.pdf

ITESM. (28 de enero de 2019). Reconocen al Tec por su contribución a la educación ambiental. Obtenido de Tecnológico de Monterrey: https://tec.mx/es/noticia s/nacional/investigacion/reconocen -altec-por-su-contribucion-la-educacion-ambiental

LEGISMEX. (7 de Octubre de 2008). Reglamento de la Ley de Residuos Sólidos del Distrito Federal. Obtenido de LEGISMEX: http://legismex.mty.itesm.mx/estados/ley-df/DF-RLeyResSol2008_12.pdf

LEGISMEX. (14 de Agosto de 2011). Reglamento de la Ley de Gestión Integral de los Residuos del Estado de Jalisco. Obtenido de LEGISMEX: http://legismex.mty.itesm.mx/estados/leyja1/JAL-R-GestIntResiduos2011_08.pdf

LEGISMEX. (28 de Abril de 2016). Reglamento para la Prevención y Gestión Integral de los Residuos del Municipio de Guadalajara. Obtenido de LEGISMEX: http://legismex.mty.itesm.mx/estados/ley-jal/JAL-RM-GdaPrevGesInRes2016_04.pdf

LEGISMEX. (28 de Abril de 2016). Reglamento para la Prevención y Gestión Integral de los Residuos del Municipio de Guadalajara. Obtenido de LEGISMEX: http://legismex.mty.itesm.mx/estados/ley-jal/JAL-RM-GdaPrevGesInRes2016_04.pdf

LEGISMEX. (26 de Abril de 2019). Ley de Residuos Solidos del Distrito Federal. Obtenido de LEGISMEX: http://legismex.mty.itesm.mx/estados/ley-df/DF-LResSol2019_04.pdf

LEGISMEX. (29 de Julio de 2019). Reglamento de la Ley Ambiental del Estado de Nuevo León. Obtenido de LEGISMEX: http://legismex.mty.itesm.mx/estados/ley-nl/NL-RLeyAmb2016_08.pdf

LYRSA. (19 de Octubre de 2015). Los Orígenes del Reciclaje. Obtenido de LYRSA Derichebourg: https://www.lyrsa.es/los-origenesdel-reciclaje/

Montes, S. (10 de Enero de 2019). Seis países alrededor del mundo reciclan más de $50 \%$ de su basura durante el año. $L a$ República. Obtenido de https://www.larepublica.co/responsabilidad-social/seis-paisesa lrededor-del-mundo-reciclan-mas-de-50-de-su-basuradurante-el-ano-2813051

Moya, V. D. (20 de Febrero de 2019). Norma Ambiental Estatal NAESDS-005-2019. Periódico Oficial, págs. 17-24. Obtenido de http://sgi.nl.gob.mx/Transparencia_2015/Archivos/AC_0001_0 007_00167319_000001.pdf

Ramírez, A. (1 de junio de 2018). 5 programas de RSE de Nestlé. Obtenido de expoknews: https://www.expoknews.com/programas-de-rse-de-nestle/

Razo, L. (7 de Junio de 2017). Premian reciclaje en las escuelas. El Diario NTR. Obtenido de https://www.ntrguadalajara.com/post.php?id_nota $=73643$

Sanmartín, G., Zhigue, R., \& Alaña, T. (2017). El Reciclaje: un nicho de innovación y emprendimiento con enfoque ambientalista. Universidad y Sociedad, 9(1), 36-40. Obtenido de http://scielo.sld.cu/pdf/rus/v9n1/rus05117.pdf

SEDEMA. (26 de Enero de 2017). Ciudad de México promueve la educación ambiental. Obtenido de Tecnologia Ambiental. 
Publicación semestral, Boletín Científico INVESTIGIUM de la Escuela Superior de Tizayuca, Vol. 6, No.11 (2020) 24-32

Sostenibilidad Empresarial:

https://tecnologiaambiental.mx/2017/01/26/educacion-

ambiental/

Sedema. (19 de Agosto de 2018). Avanza SEDEMA en la cultura del reciclaje para la CDMX. Obtenido de Avanza SEDEMA en la cultura del reciclaje para la CDMX: https://www.sedema.cdmx.gob.mx/comunicacion/nota/avanzasedema-en-la-cultura-del-reciclaje-para-la-cdmx

SEDEMA. (marzo de 2020). Centros de Cultura Ambiental. Obtenido de Gobierno de la Ciudad de México: http://www.data.sedema.cdmx.gob.mx:8081/culturaambiental/ \#

SEMARNAT. (8 de Octubre de 2003). Ley General para la Prevención y Gestión integral de Residuos. Obtenido de SEMARNAT : http://biblioteca.semarnat.gob.mx/janium/Documentos/Ciga/lib ros2009/190117.pdf

Tamayo, H. (20 de Febrero de 2018). Formemos una cultura de reciclaje en el Tecnológico de Monterrey. Obtenido de Tecnológico de Monterrey: https://tec.mx/es/noticias/ciudadjuarez/educacion/formemos-una-cultura-de-reciclaje-en-el-tec 